\title{
Cognitive behavioral therapy group intervention for HIV transmission risk behavior in perinatally infected adolescents
}

\author{
Ruth M. Senyonyi ${ }^{1}$, Lee A. Underwood ${ }^{1 *}$, Elisabeth Suarez ${ }^{1}$, Seggane Musisi ${ }^{2}$, \\ Todd L. Grande ${ }^{3}$ \\ ${ }^{1}$ School of Psychology \& Counseling, Regent University, Virginia Beach, USA; ${ }^{*}$ Corresponding Author: leeunde@regent.edu \\ ${ }^{2}$ Department of Psychiatry, Makerere University, Kampala, Uganda \\ ${ }^{3}$ College of Social and Behavioral Sciences, Wilmington University, New Castle, USA
}

Received 25 July 2012; revised 20 September 2012; accepted 30 September 2012

\section{ABSTRACT}

Purpose: This study analyzed treatment effects of cognitive behavioral therapy (CBT) group counseling intervention on HIV transmission risk behavior, depression, anxiety, and alcohol use in HIV perinatally infected adolescents attending an HIV clinical care center in Uganda, Africa. Method: A total of $\mathbf{1 8 6}$ adolescents were randomly assigned to an experimental or control arm and assigned into groups of 11 - 16 adolescents. The experimental groups received an 80-minute CBT based weekly intervention for 8 consecutive weeks, while the control groups received only standard group care. Data from self report assessments were analyzed at preand post-test using repeated measures of analysis of variance. The participants, analyzed by variable, included $\mathbf{1 1 5}$ for sexual behavior, 106 for depression, 88 for anxiety, and $\mathbf{1 1 5}$ for alcohol use. Results: The results from the study show a large significant difference $(p=0.006)$ between the experimental and control groups on the anxiety variable. There were no significant differences between the experimental and control groups on the variables of sexual behavior $(p=0.876)$, depression $(p=0.700)$, and alcohol use $(p=0.815)$. There was an indication of reduced levels of HIV sexual transmission risk behavior, depression, and alcohol use levels in the participants in both the experimental and control groups. Conclusions: Risky sexual behavior places HIV perinatally infected adolescents at risk of re-infection, sexually transmitted diseases, and transmitting HIV to their sexual partners. CBT group counseling intervention equipped the participating adolescents with appropriate life skills to deal with psychological distress and anxiety often seen in their lives and may be useful in routine medical care to reduce transmission risks and improve wellness and call for its incorporation into HIV preventive programs and counselor education.

Keywords: Group Counseling; Cognitive Behavioral Therapy; HIVIAIDS; Adolescents; Perinatally Infected

\section{INTRODUCTION}

Sub-Saharan Africa remains the region most heavily affected by HIV, and in 2008, accounted for $67 \%$ of worldwide HIV infections, $68 \%$ of new infections among adults, $91 \%$ of new infections among children, and $80 \%$ AIDS orphans [1]. In Uganda it was estimated that $1,101,317$ people were living with HIV, of these, 120,000 were children between 0 - 14 years [2].

The introduction of highly active antiretroviral therapy (HAART) has resulted in a profound decrease in HIV associated morbidity and mortality and increased the number of children who now live to adolescence and childbearing age [3]. This unique cohort of HIV perinatally infected children has grown to adolescence in the last 25 years and is considered to be at the centre of the global HIV/AIDS epidemic both in terms of new infections and opportunities for halting the transmission of HIV [3,4]. These adolescents, infected with HIV/AIDS from infancy, have sexual and procreation desires like their non-infected peers but these put them at risk for HIV re-infection and infecting their sexual partners [5]. Other factors that predispose these adolescents to further risk include psychiatric disorders, alcohol and drug use, and vulnerability as orphans $[6,7]$.

Interventions that target a reduction in transmission risk behavior increase young people's knowledge and 
that teach life skills among the HIV infected adolescents are potentially the greatest force for change because young people are more likely to adopt and maintain safe sex behavior [6]. However, relatively little empirical research exists examining interventions aimed at reducing HIV transmissions and improving the emotional well being of adolescents with HIV [8]. Other studies on reduction of sexual transmission risk behavior concentrated on HIV positive men who have sex with men, and women with HIV, and HIV infected youths but did not focus on HIV perinatally infected youth $[9,10]$. There is an intervention gap targeting HIV transmission in perinatally infected adolescents. This study, therefore, investigated the use CBT group counseling as an intervention to reduce the HIV transmission risk behavior and the associated factors of depression, anxiety and alcohol use among HIV perinatally infected adolescents in Uganda.

A basic assumption of the behavioral perspective is that most problematic behaviors, cognitions, and emotions have been learned and they can be modified by new learning. Cognitive behavioral therapy (CBT) is an educational and treatment experience where members are taught how to view their own learning process, develop a new perspective on ways of learning, and try more effective ways of changing behaviors, cognitions, and emotions [11]. CBT group therapy employs a variety of cognitive and behavioral approaches and social skills training to reduce the effects of depression, anxiety, and alcohol use [12].

To the investigator's knowledge, there were no known studies in Uganda describing the possible role of CBT group counseling intervention in reducing HIV sexual transmission risk, especially in HIV perinatally infected youth. The advantages mentioned by Elliot et al. (2004) confirm that group therapy using the CBT approach would be a feasible intervention in this study population.

\section{METHOD}

The study employed the experimental-between-groups design and utilized an experimental pretest-posttest randomized controlled trial technique. The study was conducted at Mildmay Uganda a clinical health facility that provides comprehensive services to HIV infected adults and children [13]. The study explored the effects of the CBT group counseling intervention, the independent variable IV, on the dependent variables of participant's transmission risk behavior, depression levels, anxiety levels and alcohol use rates.

\subsection{Sampling and Study Participants}

The study participants were all Ugandan adolescents between the ages of 12 - 18 years that acquired HIV through transmission from an infected mother during the prenatal or peri-partum period or were infected by HIV at birth, were recruited by convenience sampling, and were presently receiving care from Mildmay Uganda. Out of the 328 adolescents contacted by phone and/or face to face, 186 adolescents fit the inclusion criteria, agreed to participate, and were recruited into the study. They were randomly assigned into one of the two arms (experimental and control). Of the 186 participants, 15 $(8 \%)$ were eliminated, leaving a total of 171 (92\%) participants registered as attendee participants in the study. Of the 171 attendee participants, the final participants included only those that had attended at least three sessions and had completed one or more of the four assessing instruments at both pre- and post-test. The number of participants varied across the four dependent variables and the final analyses included 115 for sexual behavior, 106 for depression (CDI), 88 for anxiety (RCMAS), and 115 for alcohol use (AUDIT). See Table 1.

There were no significant differences $(p>0.05)$ found across the dependant variables between the experimental and control groups in terms of gender, age, education levels, number of years on ARV's and types of schools attended. Tables 2-5 indicate the detailed demographics of participants enrolled in the different assessments of sexual behavior, depression, anxiety and alcohol use respectively.

Overall, for all the assessments, the baseline analysis found that the experimental groups and control groups were relatively similar in terms of the demographics collected, and found no significant differences between the participants in the experimental arm and the control arm that could have affected the results of study.

\subsection{Assessment Instruments}

The study instruments (self reports) consisted of: 1) The Social Demographic Questionnaire used to assess age, gender, education, school details, ethnicity/tribe, ditrict, start on ART, family history, orphan-hood status,

Table 1. Number of participants included in the analyses per assessment.

\begin{tabular}{cccc}
\hline \multirow{2}{*}{ Assessment } & \multicolumn{3}{c}{ Completed Assessments (Pre- \& Posttests) } \\
\cline { 2 - 4 } & Experimental arm & Control arm & Total \\
\hline Sexual Behavior & 80 & 35 & 115 \\
CDI & 74 & 32 & 106 \\
RCMAS-2 & 63 & 25 & 88 \\
AUDIT & 81 & 34 & 115 \\
\hline
\end{tabular}

Note. CDI = Child Depression Inventory; RCMAS-2 = Revised Children's Manifest Anxiety; Scale-2 ${ }^{\text {nd }}$ ed.; AUDIT = Alcohol User Disorder Identification Scale. 
Table 2. Baseline demographic characteristics study participants for the sexual behavior assessment $(\mathrm{n}=115)$.

\begin{tabular}{|c|c|c|c|c|c|}
\hline \multirow{2}{*}{ Characteristic } & \multicolumn{2}{|c|}{ Group M (SD) } & \multirow{2}{*}{ Total sample M $(S D)[\mathrm{n} \%]$} & \multirow{2}{*}{$F\left(X^{2}\right)$} & \multirow{2}{*}{$p$} \\
\hline & Intervention & Control & & & \\
\hline Age & $15.19(1.93)$ & $15.06(1.98)$ & $15.15(1.94)$ & 0.11 & 0.7 \\
\hline Years on ARVs & $11.27(2.59)$ & $10.96(2.86)$ & $11.17(2.67)$ & 0.25 & 0.6 \\
\hline Gender & & & & $(0.40)$ & 0.5 \\
\hline Male & 36 & 18 & 54 [46.9] & & \\
\hline Female & 44 & 17 & $61[53]$ & & \\
\hline Total & 80 & 35 & 115 & & \\
\hline Education & & & & $(18.88)$ & 0.2 \\
\hline Primary & 30 & 16 & $45[39.2]$ & & \\
\hline O Level Secondary & 33 & 13 & $46[40.1]$ & & \\
\hline A Level Secondary & 12 & 02 & 14 [12.2] & & \\
\hline Postsecondary (Course/Institute) & 04 & 02 & $06[5.2]$ & & \\
\hline Self-employed & 00 & 01 & $01[0.9]$ & & \\
\hline None & 02 & 01 & $03[2.6]$ & & \\
\hline Total & 80 & 35 & 115 & & \\
\hline Type of School & & & & $(11.24)$ & 0.4 \\
\hline Day & 43 & 13 & $56[48.7]$ & & \\
\hline Boarding & 06 & 04 & $10[8.7]$ & & \\
\hline Other (Girls/Boys/Mixed) & 24 & 13 & $37[32.1]$ & & \\
\hline N/A & 07 & 05 & $12[10.5]$ & & \\
\hline Total & 80 & 35 & 115 & & \\
\hline Tribe & & & & $(17.81)$ & 0.4 \\
\hline Baganda & 55 & 27 & 82 [71.3] & & \\
\hline Bakiga/Banyankole & 10 & 01 & $11[9.5]$ & & \\
\hline Other & 15 & 07 & 22 [19.2] & & \\
\hline Total & 80 & 35 & 115 & & \\
\hline
\end{tabular}

Note: ARV = Antiretroviral Drugs; O Level Secondary = Ordinary Level Secondary; A Level Secondary = Advanced Level Secondary.

place of residence, and marital status; 2) Sexual History Questionnaire by California Action Coalition was used to obtain sexual history data of the last one year. The total sexual behavior score used in the analyses was obtained on number of recent sexual encounters, sexual partners, vaginal and anal sexual encounters and use of condoms. The post-intervention questionnaire was adjusted to request for sexual history information of the previous two months. The Cronbach's alpha was calculated at 0.88 ; 3) Child Depression Inventory [14] was used to assess depression. The total score, considered for this study, indicated overall depressive symptomatology across the five sub-scales namely: negative mood, interpersonal difficulties, negative self-esteem, ineffectiveness, and anhedonia [14]. The internal consistency coefficients range from 0.71 to 0.89 , and the test-retest coefficient range from 0.74 to 0.83 [12]. Cronbach's alpha reliability was calculated at 0.87 for pre-CDI and 0.86 for post-CDI; 4) Revised Children's Manifest Anxiety Scale-Second Edition (RCMAS-2) [15] measured the level and nature of anxiety. The total anxiety score, used in this study, was based on physiological, worry, and social anxiety. Instrument reliability (Cronbach's Alpha) for the pre-RCMAS was calculated at 0.88 and was 0.87 at post-RCMAS; 5) The Alcohol Use Disorders Identification Test (AUDIT), was used to screen for alcohol use, recent alcohol dependence symptoms, and alcohol related problems [16]. AUDIT indicates high internal consistency with a test-retest reliability score of 0.86 and was found to be an accurate measure of risk across 
Table 3. Baseline demographic characteristics of study participants for the CDI $(n=106)$.

\begin{tabular}{|c|c|c|c|c|c|}
\hline \multirow{2}{*}{ Characteristic } & \multicolumn{2}{|c|}{ Group M $(S D)$} & \multirow{2}{*}{ Total sample M $(S D)[\mathrm{n} \%]$} & \multirow{2}{*}{$F\left(X^{2}\right)$} & \multirow{2}{*}{$p$} \\
\hline & Intervention & Control & & & \\
\hline Age & $15.30(1.95)$ & $15.03(2.07)$ & 15.22 & 0.40 & 0.53 \\
\hline Years on ARVs & $11.45(2.68)$ & $10.84(2.93)$ & 11.26 & 0.84 & 0.36 \\
\hline Gender & & & & $(0.59)$ & 0.44 \\
\hline Male & 31 & 16 & $47[44.3]$ & & \\
\hline Female & 43 & 16 & $59[55.6]$ & & \\
\hline Total & 74 & 32 & 106 & & \\
\hline Education & & & & $(23.19)$ & 0.08 \\
\hline Primary & 25 & 15 & 40 [37.7] & & \\
\hline O Level Secondary & 33 & 14 & $41[38.6]$ & & \\
\hline A Level Secondary & 13 & 03 & $15[14.1]$ & & \\
\hline Postsecondary (Course/Institute) & 04 & 02 & $05[4.7]$ & & \\
\hline Self-employed & 00 & 01 & $01[0.9]$ & & \\
\hline None & 03 & 01 & $04[3.8]$ & & \\
\hline Total & 74 & 32 & 106 & & \\
\hline Type of School & & & & $(7.95)$ & 0.72 \\
\hline Day & 40 & 14 & $54[50.9]$ & & \\
\hline Boarding & 05 & 03 & 08 [7.5] & & \\
\hline Other (Girls/Boys/Mixed) & 22 & 11 & $33[31.1]$ & & \\
\hline N/A & 07 & 04 & $11[10.1]$ & & \\
\hline Total & 74 & 32 & 106 & & \\
\hline Tribe & & & & $(20.86)$ & 0.29 \\
\hline Baganda & 52 & 26 & $78[73.6]$ & & \\
\hline Bakiga/Banyankole & 09 & 00 & $09[8.5]$ & & \\
\hline Other & 13 & 06 & 19 [17.9] & & \\
\hline Total & 74 & 32 & 106 & & \\
\hline
\end{tabular}

Note: ARV = Antiretroviral Drugs; O Level Secondary = Ordinary Level Secondary; A Level Secondary = Advanced Level Secondary.

gender, age, and culture [14]. The reliability for the preAUDIT was calculated at 0.85 and was 0.86 at postAUDIT.

\subsection{Intervention}

The intervention was conducted using Teens Linked to Care (2001), Module II-Act Safe and utilized group counseling based on CBT approaches. Teens Linked to Care (TLC) is an intervention for young people living with HIV, based on behavioral and social science theory, and delivered in small groups using cognitive-behavioral strategies to change behavior [17]. The Act Safe Module intervention aimed to reduce sexual transmission acts and substance use by having youths identify their risk behavior triggers and modify their patterns of substance use as well as increase self efficacy of condom use [17]. The participants in the experimental groups, led by trained counselors, were randomly assigned to the experimental group, comprised of eight groups with approximately $13-18$ adolescents in each group and were given 80-minute weekly CBT group counseling on eight consecutive Saturday afternoons. The intervention utilized group counseling based on CBT approaches most applicable to HIV-infected persons and addressed the following issues: psycho-education and information about HIV/AIDS, self-knowledge and self-disclosure skills, problem solving, self-efficacy, anxiety, depression, and sexual transmission risks related to HIV/AIDS. The intervention encouraged sexual abstinence using the True Love Waits [18] information and commitment cards. The True Love Waits program encourages adolescents to 
Table 4. Baseline demographic characteristics study participants for the RCMAS-2 $(n=88)$.

\begin{tabular}{|c|c|c|c|c|c|}
\hline \multirow{2}{*}{ Characteristic } & \multicolumn{2}{|c|}{ Group M $(S D)$} & \multirow{2}{*}{ Total sample M $(S D)[\mathrm{n} \%]$} & \multirow{2}{*}{$F\left(X^{2}\right)$} & \multirow{2}{*}{$p$} \\
\hline & Intervention & Control & & & \\
\hline Age & $15.46(1.89)$ & $15.32(2.16)$ & $15.42(1.96)$ & 0.09 & 0.76 \\
\hline Years on ARVs & $11.62(2.53)$ & $10.50(3.43)$ & $11.25(2.88)$ & 2.28 & 0.14 \\
\hline Gender & & & & (.19) & 0.66 \\
\hline Male & 27 & 12 & $39[44.3]$ & & \\
\hline Female & 36 & 13 & $49[55.7]$ & & \\
\hline Total & 63 & 25 & 88 & & \\
\hline Education & & & & $(20.82)$ & 0.14 \\
\hline Primary & 19 & 10 & $29[33.0]$ & & \\
\hline O Level Secondary & 27 & 10 & $37[42.1]$ & & \\
\hline A Level Secondary & 12 & 02 & 14 [15.9] & & \\
\hline Postsecondary (Course/Institute) & 03 & 01 & $04[4.5]$ & & \\
\hline Self-employed & 02 & 01 & $01[1.1]$ & & \\
\hline None & 63 & 25 & $03[3.4]$ & & \\
\hline Total & & & 115 & & \\
\hline Type of School & & & & $(7.19)$ & 0.71 \\
\hline Day & 33 & 10 & $43[71.6]$ & & \\
\hline Boarding & 05 & 03 & $08[9.1]$ & & \\
\hline Other (Girls/Boys/Mixed) & 19 & 09 & $28[31.7]$ & & \\
\hline N/A & 06 & 03 & $09[10.0]$ & & \\
\hline Total & 63 & 25 & 88 & & \\
\hline Tribe & & & & $(17.02)$ & 0.32 \\
\hline Baganda & 44 & 19 & $63[69.6]$ & & \\
\hline Bakiga/Banyankole & 00 & 09 & $09[10.2]$ & & \\
\hline Other & 10 & 06 & 16 [17.9] & & \\
\hline Total & 63 & 25 & 88 & & \\
\hline
\end{tabular}

Note: ARV = Antiretroviral Drugs; O level Secondary = Ordinary Level Secondary; A Level Secondary = Advanced Level Secondary.

commit to sexual abstinence until marriage by adhering to biblical principles. The intervention schedule included in the study is outlined in Table 6.

\subsection{Control Group}

The participants in the control group received standard group counseling for 8 consecutive weeks and were eligible to receive intervention at the end of the study. The participants randomized into the control arm comprised of four groups with approximately $13-18$ adolescents in each group. Each group was assigned to a trained counselor, randomly assigned to groups at the beginning of the study. The leaders were informed that the participants would receive standard group care. These groups met at the same site but in a different building from the inter- vention arm. The control arm counselors met separately for an hour before and an hour after the sessions for planning and debriefing. A number of standard care topics were discussed that included disclosure of HIV status to members at home and at school, ARVs drug adherence, and self-care.

Intervention and control attendees were those respectively assigned to the intervention or control arms, attended at least three sessions, and completed the pre- and post-assessments. Participants who missed more than three sessions were excluded from the findings of the study.

\subsection{Ethical Considerations}

Ethical clearance was obtained from the Human Subjects 
Table 5. Baseline demographic characteristics study participants for the audit $(n=115)$.

\begin{tabular}{|c|c|c|c|c|c|}
\hline \multirow[b]{2}{*}{ Characteristic } & \multicolumn{2}{|c|}{ Group M $(S D)$} & \multirow{2}{*}{ Total sample M $(S D)[\mathrm{n} \%]$} & \multirow{2}{*}{$F\left(X^{2}\right)$} & \multirow{2}{*}{$p$} \\
\hline & Intervention & Control & & & \\
\hline Age & $15.19(1.96)$ & $15.38(1.97)$ & $15.24(1.96)$ & 0.24 & 0.62 \\
\hline Years on ARVs & $11.62(2.53)$ & $10.50(3.43)$ & $11.25(2.88)$ & 1.13 & 0.29 \\
\hline Gender & & & & $(0.06)$ & 0.79 \\
\hline Male & 36 & 16 & $52[45.2]$ & & \\
\hline Female & 45 & 18 & $63[54.8]$ & & \\
\hline Total & 81 & 34 & 88 & & \\
\hline Education & & & & $(20.72)$ & 0.15 \\
\hline Primary & 29 & 14 & $43[37.5]$ & & \\
\hline O Level Secondary & 33 & 13 & $46[40.0]$ & & \\
\hline A Level Secondary & 13 & 03 & 16 [13.9] & & \\
\hline Postsecondary (Course/Institute) & 03 & 02 & $05[4.4]$ & & \\
\hline Self-employed & 00 & 01 & $01[0.9]$ & & \\
\hline None & 03 & 01 & $04[3.5]$ & & \\
\hline Total & 81 & 34 & 115 & & \\
\hline Type of School & & & & $(7.98)$ & 0.15 \\
\hline Day & 43 & 13 & $56[48.7]$ & & \\
\hline Boarding & 06 & 03 & $09[7.8]$ & & \\
\hline Other (Girls/Boys/Mixed) & 24 & 14 & $38[31.1]$ & & \\
\hline N/A & 08 & 04 & $11[10.1]$ & & \\
\hline Total & 81 & 34 & 115 & & \\
\hline Tribe & & & & $(20.62)$ & 0.63 \\
\hline Baganda & 55 & 25 & $80[69.6]$ & & \\
\hline Bakiga/Banyankole & 11 & 01 & $12[10.4]$ & & \\
\hline Other & 15 & 08 & $23[20]$ & & \\
\hline Total & 81 & 34 & 115 & & \\
\hline
\end{tabular}

Note: ARV = Antiretroviral Drugs; O level Secondary = Ordinary Level Secondary; A Level Secondary = Advanced Level Secondary.

Review Committee at Regent University, from Mildmay Uganda Research Ethics Committee and from the Uganda National Council of Science and Technology. Assent and consent was received from the participants, and parents or caretakers.

\subsection{Statistical Analyses and Hypothesis}

Data were collected from participants using self-report assessments at baseline and at the end of the intervention. The raw data was coded and transferred to Excel spreadsheets and later to the SPSS program for analysis. All participants received a total score at baseline and at post intervention on the assessments. The mean scores and standard deviations at baseline and post intervention were compared for any significant changes. Repeated measures of ANOVA statistical tests were used to address the main hypotheses: that HIV perinatally infected adolescents at Mildmay Uganda who received the CBT group counseling intervention would show significantly less HIV transmission risk associated sexual behavior, and lower levels on the associated factors of depression, anxiety and alcohol use when compared to the control standard care group at post test. To address missing items in the CDI and RCMAS assessments, average mean substitution was done except for cases where three or more responses were incomplete. The video and audio tapes were analyzed for procedural adherence and content, and observed for any other information related to the study. 
Table 6. Intervention schedule.

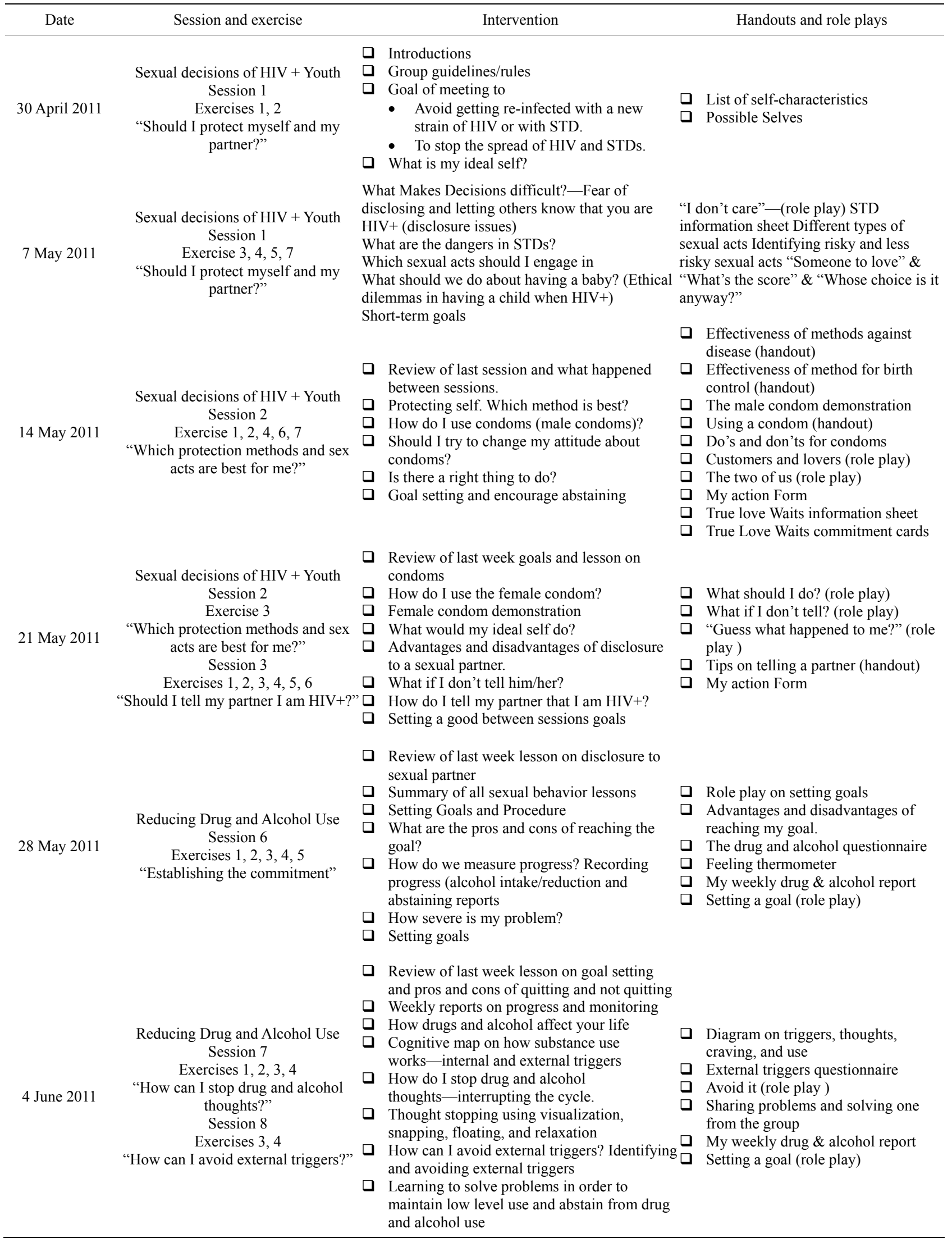




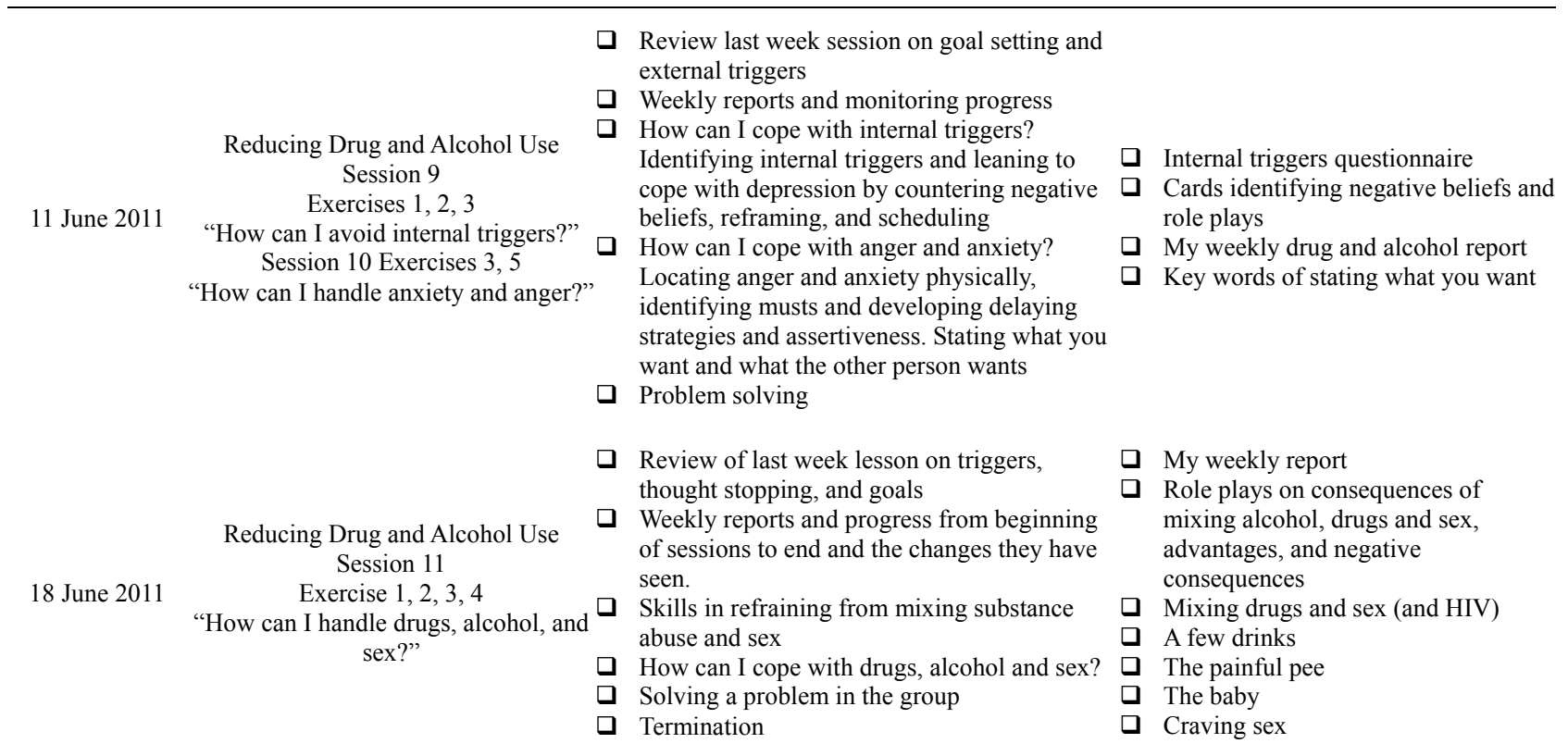

\section{RESULTS}

Participants eligible for analysis at the end of the study were only those that had attended at least three sessions and had completed pre- and posttests in any of the four assessments. Out of the 171 who attended the study, four participants completed only the demographic information but none of the assessments at either pre- or post-test. Six participants completed posttest assessments but did not complete any assessments at pretest. Forty participants completed the assessments at pretest but did not complete any assessments at posttest. That left 121 participants with usable data. Attendance during the 8 weeks of intervention varied between 104 - 150 children per week. The participant attrition, therefore, appeared to be unrelated to the participants' scores on the measures used in this study. Thus, the likelihood of attrition for nonrandom reasons adversely impacting the findings of the study did not appear to be a major threat to the internal validity of the data. The number of participants varied across the four dependent variables and therefore each variable, sexual transmission, depression, anxiety and alcohol use, was analyzed by separately. Thus the data analyzed for the sexual behavior assessment were 115 participants; the CDI assessment included 106 participants; the RCMAS assessment included 88 participants; and the AUDIT assessment included 115 participants (Table 1). There were no important differences among the two groups (experimental and control) across all of the variables at baseline that could have influenced the study Thus randomization process appeared to have been effective. The results of model assumptions of normality, homogeneity of covariance and independence of obser- vations were satisfactory. Table 7 summarizes the means, standard deviations and repeated measures ANOVA results of the CBT group intervention effect based on comparisons of the experimental and control groups.

\subsection{Impact of CBT Group Intervention on Sexual Behavior}

The investigator hypothesized that HIV perinatally infected adolescents who received the CBT group counseling intervention would show significantly less HIV transmission risk associated sexual behavior as indicated by self-report outcomes in the following variables at posttest compared to those in the control group: less number of sexual encounters (intercourse or penetrative sex), less number of sexual partners, and less unprotected penile penetrative vaginal sexual acts (intercourse; i.e., use of condoms, and continued abstinence). The sexual behavior analysis was conducted using 115 participants. The results of total sexual behavior score expressed as mean scores for the experimental arm, showed a decrease in HIV transmission risk associated sexual behavior at two months posttest, $2.11(S D=2.75)$ to $1.24(S D=1.40)$ while the control arm scores also indicated a decrease from $1.83(S D=2.57)$ to $1.11(S D=0.68)$. The results indicated a decrease in the total score for sexual transmission behavior for both the experimental and control groups, Wilk's $\lambda=0.951, F(1,113)=5.866, p=0.017$, partial $\eta^{2}=0.049$. A repeated measures ANOVA, however, showed no significant group differences on the dependent variable (sexual behavior) when compared to the control group at posttest, Wilk's $\lambda=1.00, F(1,113)=$ $0.024, p=0.876$, partial $\eta^{2} \leq 0.001$. 
Table 7. Means, standard deviations and repeated measures ANOVA results for the time effect and time $\mathrm{x}$ group effect of CBT intervention on study participants.

\begin{tabular}{cccccccc}
\hline \multirow{2}{*}{ Variable } & \multicolumn{4}{c}{ Group M $(S D)$} & \multirow{2}{c}{$\begin{array}{c}\text { Time x Effect (Within Groups } \\
\text { Comparison) }\end{array}$} & $\begin{array}{c}\text { Time x Group Effect (Between } \\
\text { Groups Comparison) }\end{array}$ \\
\cline { 2 - 4 } & \multicolumn{2}{c}{ Experimental } & \multicolumn{2}{c}{ Control } & & $F(p)$ \\
Pre-test & Post-test & Pre-test & Post-test & & $F(p)$ \\
Sexual Behavior & $2.1(2.8)$ & $1.2(1.4)$ & $1.8(2.6)$ & $1.1(0.7)$ & $5.9(0.02)^{*}$ & $0.02(0.88)$ \\
Depression & $38.9(7.9)$ & $36.1(6.7)$ & $39.4(10.0)$ & $37.3(9.9)$ & $10.0(0.002)^{*}$ & $0.2(0.7)$ \\
Anxiety & $61.2(6.9)$ & $64.5^{1}(7.9)$ & $64.4(7.3)$ & $62.6(7.6)$ & $0.6(0.43)$ & $8.1(0.06)^{*}$ \\
Alcohol Use & $0.69(2.7)$ & $0.3(1.6)$ & $0.9(3.8)$ & $0.5(2.6)$ & $5.4(0.02)^{*}$ & $0.1(0.82)$ \\
\hline
\end{tabular}

${ }^{1} \mathrm{An}$ increase in means indicates decrease in anxiety; ${ }^{*}$ Significant at $p<0.05$ level.

\subsection{Impact of CBT Group Intervention on Depression}

The investigator hypothesized that HIV perinatally infected adolescents who received the CBT group counseling intervention would show significantly lower levels of depression, as measured by the analysis on depression was conducted using 106 participants. The total CDI score expressed as mean scores for the experimental group showed a decrease in depression at 2 months posttest, $38.85(S D=7.87)$ to $36.05(S D=6.67)$, while the control group scores also showed a decrease in depression from $39.44(S D=10.09)$ to $37.26(S D=9.85)$. The results indicated a decrease in depression in both the experimental and control groups, Wilk's $\lambda=0.912, F(1$, $104)=10.027, p=0.002$, partial $\eta^{2}=0.088$. A repeated measures ANOVA, however, showed no significant group differences on the dependent variable (depression) when compared to the control group at post test, Wilk's $\lambda$ $=0.999, F(1,104)=0.149, p=0.700$, partial $\eta^{2}=0.001$

(Table 3).

\subsection{Impact of CBT Group Intervention on Anxiety}

The investigator hypothesized that HIV perinatally infected adolescents who received the CBT group counseling intervention would show significantly lower levels of anxiety, as measured by the total score on the RCMAS-2, at posttest when compared to the control group. The analysis on anxiety was conducted using 88 participants. The results of the total anxiety score were expressed as mean scores (where an increase in the means indicated a decrease in anxiety). The experimental group, showed a significant decrease in anxiety at two months posttest, $\mathrm{M}=61.22(S D=6.85)$ to $64.46(S D=$ 7.90) while the control group scores showed a mild increase in anxiety from $64.39(S D=7.34)$ to $62.55(S D=$ 7.63). The mean scores in the experimental group showed a significant decrease in anxiety over time relative to the control group. A repeated measures ANOVA indicated a very large significant group difference on the dependent variable (anxiety) when compared to the control group at posttest, Wilk's $\lambda=0.914, F(1,86)=8.095$, $p=0.006$, partial $\eta^{2}=0.086$.

\subsection{Impact of CBT Group Counseling on Alcohol Use}

The investigator hypothesized that HIV perinatally infected adolescents who received the CBT group counseling intervention would show significantly less instances of alcohol use, as measured by the total score on the AUDIT, at posttest when compared to the control group. The analysis on alcohol use was conducted using 115 participants. The results indicated by the total AUDIT score and expressed as mean scores for the experimental group showed a decrease in alcohol use at two months posttest $0.69(S D=2.73)$ to $0.26(S D=1.55)$, and the control group scores also showed a decrease in alcohol intake from $0.82(S D=3.82)$ to $0.47(S D=2.57)$. The results indicated a decrease in alcohol levels for both groups, Wilk's $\lambda=0.955, F(1,113)=5.386, p=0.022$, partial $\eta^{2}=0.045$. A repeated measures ANOVA showed no significant group differences on the dependent variable (alcohol use), when compared to the control group at posttest, Wilk's $\lambda=1.00, F(1,113)=0.055, p=0.815$, partial $\eta^{2}<0.001$.

\section{DISCUSSION}

The results on sexual risk behavior, when compared at pre-and posttest, indicated a decrease in risky sexual behavior in all groups. Of the 115 participants, 17 (15\%) who had reported current sexual behavior at baseline, showed no sexual activity at posttest. Six who had sex without condoms at pretest reported no longer having sex at posttest. Ten who had reported having sex with condoms at pre test reported no longer having sex at posttest. Two had reported having sex at pretest started to use condoms at post test. Two participants who had no sex at pretest became sexually active by the end of the study but reported using condoms all the time. Given the scarcity of interventions for HIV transmission behavior 
among HIV perinatally infected adolescents, the reduction in risky behavior in this study demonstrate a need for similar effective interventions to lower risk behavior.

The study registered a high rate of abstinence with 97 participants $(83 \%)$ at baseline who remained abstaining at post intervention. This is not surprising given that our sample were still rather young with a mean age of 15.15 and with $39.2 \%$ in primary school. This is supported by the Uganda Demographic and Health Survey, 2006, which indicated that the median age at first sex for women ages 20 - 49 is 16.6 years [19]. During the study, participants in the experimental group were encouraged to abstain from sex. In addition, the participants had, for several years, been attending this health facility-Mildmay Uganda - that encourages abstinence until marriage. This indicates the importance of abstinence, an important objective in preventive interventions with HIV-positive adolescents. The reports of abstinence need to be followed up in further studies to observe their sustainability.

The low rates of sexual risk activity among the participants at baseline (15\%) may suggest a possible delay of onset of sexual activity among the HIV perinatally infected adolescents. This is consistent with clinical findings in a study [20], where HIV perinatally infected children were identified as slow progressors with stunted growth accounting for the delay in onset of sexual activity. Other studies identified other causes of delay as slow pubertal development, cognitive deficits, multiple hospitalizations, losses, and stressors that complicate decisions related to HIV disclosure to partners [21,22]. Since the present study only explored penetrative (vaginal and anal) sexual behaviors, further studies can concurrently assess the different risky HIV sexual transmission behaviors to better inform HIV preventive programs.

The information provided using the CBT approach in group counseling in the present study was timely and relevant to the HIV positive adolescents and will be useful in helping them make life time decisions for self-preservation. The CBT group counseling approach therefore needs to be widely disseminated in Uganda. This study confirms that HIV positive adolescents need structured group support services where they meet each other to receive peer support, life skills education and psychosocial support to gain knowledge and make informed decisions [6].

Depression levels were decreased in all the groups in this study. The lowered levels of depression in the group that received CBT Group counseling can be attributed to the skills and information on handling depression provided to the intervention group. Further studies are however needed to follow up on the sustainability of the lowered depression levels similarly observed in the con- trol group that was not given the CBT skills.

Anxiety in the participants was documented from group discussions that identified fear of death whenever they were ill whilst others feared losing their parents or caretakers. It was noted that at baseline, a large number of participants had already lost their parents $(61 \%$ had lost a father and $69 \%$ had lost a mother). In addition, more anxiety within the groups was observed when one participant died during the period of the study. The statistical analysis for the participants who completed the RCMAS-2 assessment $(n=88)$ indicated no significant differences between the experimental and control groups that could have affected the results of the study. The CBT group intervention provided the participants in the experimental groups with specific practical skills in handling anxiety that enabled the participants to verbally express and deal with fears and anxieties. These are outlined in the intervention schedule Table 7. This led to a remarkably significant decrease of anxiety in the intervention group $(p=0.006)$. HIV positive adolescents can greatly benefit from CBT group counseling to acquire appropriate skills to handle anxiety in the different situations of life.

In the present study, low rates of alcohol use in the HIV perinatally infected adolescents was found at baseline where $3(2.6 \%)$ participants indicated alcohol use and $112(97.4 \%)$ participants out of the 115 indicated not using alcohol. The low rate of alcohol use in this cohort is consistent with a study on emotional and behavioral disorders in HIV positive adolescents in urban Uganda that reported only 2 out of 82 participants who used alcohol [23]. Most other studies conducted have mainly focused on adolescents identified with alcohol or substance use disorders linking them to risky sexual activity and greater numbers of sexual partners [24,25].

The positive outcome in this study was that despite the low rates of alcohol use among the participants, there was a slight (but not statistically significant) decrease in the numbers and frequency of alcohol use in those $2.6 \%$ who indicated alcohol use. For example one participant who reported drinking 2 - 3 times a week at pre test, was not drinking at all at post test, while another reduced alcohol intake from drinking daily at pre test to drinking once a week at post test. In both groups however many participants mentioned that they had observed many of their peers at school drinking alcohol and taking drugswhich meant that they too were vulnerable even though many had not started taking alcohol or drugs. In this study, abstinence from drugs and alcohol was emphasized in the intervention groups. The exercises in the CBT intervention included effects of drugs and alcohol on health, interrupting alcohol thoughts, and refraining from mixing alcohol, drugs and sex. The information provided in the study will therefore be useful in helping the adolescents abstain from alcohol use. A follow-up of 
the participants is needed to ascertain the sustainability of this alcohol abstinence emphasis. Further studies with an emphasis on the HIV perinatally infected adolescents that use or abuse alcohol needs to be done.

The strengths of this study were in the use of a randomized trial, an intervention manual and a control group as a comparison to the intervention group. The use of group counseling provided participants with peer support and psychosocial support. Cognitive behavioral approaches helped the participants focus on thoughts, feelings and behavior and provided practical and effective methods of changing cognitions, emotions and behavior.

The limitations were identified as follows: 1) The self-report assessments prompted translation into the local language and could have affected the confidentiality of the responses; 2) The intervention was carried out at the same venue with the control groups which could have confounded the results; 3) The instruments used had a satisfactory internal reliability but it was not possible to validate them; 4) Irregular attendance and attrition was observed especially at the beginning of school term and due to political riots at the time; 5) The use of a clinical sample may limit generalizability of the findings to adolescents who are not in a structured clinical environment or who are not undergoing treatment.

\section{Implications for Mental Health Providers}

Providers are encouraged to broaden and add depth to their counseling in order to cater for the many related psychosocial problems such as loss, grief, depression anxiety, stress and alcohol use in this cohort. The CBT group counseling has capacity to accommodate large client numbers and would be particularly helpful in the Uganda where the counselor-client ratio is still low. Group counseling should especially be encouraged as a peer support mechanism that allows the adolescents to discuss their fears and anxieties in group settings for maximum support. From the present study it is evident that CBT group counseling model may need to be incorporated into counselor education as a focus on prevention with positives counseling, especially targeting HIV perinatally infected adolescents.

Further research should investigate potential counseling related strategies for maintaining abstinence, safer sex practices and sexual behavior change. A replication of the study can also be done but with modifications such as a wait-list control group, a tailor-made manual specifically for the Ugandan HIV positive adolescent population and the use of a more generalized adolescent population.

\section{CONCLUSION}

This study becomes the first in Uganda to describe the role of CBT group counseling in reducing HIV transmission risk behavior and associated factors of depression, anxiety and alcohol use among HIV perinatally infected adolescents. The study is a contribution to the Uganda National AIDS Policy that seeks to prevent the transmission of HIV in Uganda. The study has shown that HIV perinatally infected adolescents, when given the right intervention have a potential to change behavior. The CBT group counseling provided the adolescents in the study with life skills to deal with psychological distress of depression and anxiety so often identified in the lives of this unique cohort that deals with the reality of HIV and the many challenges that come with the disease and with adolescence. The study encouraged sexual and alcohol abstinence and delay of onset of sexual activity among those who were abstaining and gave appropriate skills in the reduction of HIV risky transmission.

\section{REFERENCES}

[1] Joint United Nations Program on HIV/AIDS, World Health Organization (2010) Global report: UNAIDS report on the global AIDS epidemic 2010. http://www.unaids.org/globalreport/Global_report.htm.

[2] Uganda (2010) United National General Assembly special session on HIV. Country Progress Report, Kampala.

[3] Ezeanolue, E.E., Wodi, A.P., Patel, R. et al., (2006) Sexual behaviors and procreational intentions of adolescents and young adults with perinatally acquired human immunodeficiency virus infection: Experience of an urban tertiary center. Journal Adolescent Health, 38, 719-725. doi:10.1016/j.jadohealth.2005.06.015

[4] Bakeera-Kitaka, S., Nabukeera-Barungi, N., Nostlinger, C., et al. (2008) Sexual risk reduction needs of adolescents living with HIV in a clinical care setting. AIDS Care, 20, 426-433. doi:10.1080/09540120701867099

[5] Monasch, R. and Mahy, M. (2006) Young people: The centre of the HIV epidemic. In: Ross, D.A., Dick, B. and Ferguson, J., Eds., Preventing HIVIAIDS in Young People: A Systematic Review of the Evidence from Developing Countries: WHO Technical Report Series. UNAIDS Interagency Task Team on Young People, World Health Organization, Geneva, 15-41.

[6] Birungi, H., Mugisha, J.F., et al. (2008) Sexual and reproductive health needs of adolescents perinatally infected with HIV in Uganda. Population Council, Washington.

[7] Musisi, S., Kinyanda, E., Nakasujja, N., et al. (2007) A comparison of the behavioral and emotional disorders of primary school-going orphans and non-orphans in Uganda. Africa Health Science, 7, 202-213.

[8] Nicolaou, D.C. (2007) Secondary prevention for HIVpositive adolescents: Psychosocial functioning, health promoting factors, and disease control. http://idea.library.drexel.edu/bitstream/1860/1786/1/Nicol aou Despina.pdf

[9] Wingwood, G., DiClemente, R.J., Mikhail, I., et al. (2004) A randomized controlled trail to reduce HIV transmission 
risk behaviors and sexually transmitted diseases among women living with HIV: The willow program. Journal of Acquired Immune Deficiency Syndromes, 37, 58-67. doi:10.1097/01.qai.0000140603.57478.a9

[10] Choi, K.H., Lew, S., Vittinghoff, E., et al. (1996) The efficacy of brief group counseling in HIV risk reduction among homosexual Asian and Pacific Islander men. AIDS, 10, 81-87. doi:10.1097/00002030-199601000-00012

[11] Corey, G. (2000) Theory and practice of group counseling. Brooks Cole, Belmont.

[12] Rice, A.H. (2004) Group treatment of depression. In: DeLucia-Waack, D.A., et al., Eds., Handbook of Group Counseling \& Psychotherapy, Sage, Thousand Oaks, 532-546.

[13] Mildmay Uganda (2012). http://www.mildmay.org.uk/UgandaCentre.html

[14] Kovacs, M. (2003) Children's depression inventory: Technical manual update. Multi-Health Systems, North Tonawanda.

[15] Reynolds, C.R. and Richmond, B.O. (2008) Revised children's manifest anxiety scale. 7th Edition, Western Psychological Services, Los Angeles.

[16] Babor, T.F., Higgins-Biddle, J.C., Saunders, J.B., et al. (2001) The alcohol use disorders identification test: Guidelines for use in primary care. 2nd Edition, World Health Organization, Geneva.

[17] Rotherum-Borus, M.J., Lee, M.B., Birnbaum, J.M., et al. (2001) Efficacy of a preventive intervention for youths living with HIV. American Journal of Public Health, 91, 400-405. doi:10.2105/AJPH.91.3.400

[18] LifeWay (2001-2011) True love waits.
http://www.lifeway.com/ArticleView?storeId=10054\&cat

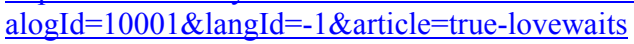

[19] Uganda Bureau of Statistics (UBOS) and Macro International, Inc. (2007) Uganda demographic and health survey 2006. UBOS and Macro International, Calverton.

[20] Bakeera-Kitaka, S., McKellar, M., Snider, C., et al. (2008) Antiretroviral therapy for HIV-1 infected adolescents in Uganda: Assessing the impact on growth and sexual maturation. The Journal of the Pediatric Infectious Diseases Society, 3, 97-104.

[21] Bauermeister, J.A., Elkington, K., Brackis-Cott, E., et al. (2009) Sexual behavior and perceived peer norms: Comparing perinatally HIV-infected and HIV-affected youth. Journal of Youth Adolescents, 38, 1110-1122. doi:10.1007/s10964-008-9315-6

[22] Brogly, S.B., Watts, D.H., Ylitalo, N., et al. (2007) Reproductive health of adolescent girls perinatally infected with HIV. American Journal of Public Health, 97, 10471052. doi:10.2105/AJPH.2005.071910

[23] Musisi, S. and Kinyanda, E. (2009) The emotional and behavioral disorders in HIV seropositive adolescents in urban Uganda. East African Medical Journal, 86, 16-24. doi:10.4314/eamj.v86i1.46923

[24] Bailey, S.L., Pollock, N.K., Martin, C.S., et al. (1999) Risky sexual behaviors among adolescents with alcohol use disorders. Journal Adolescent Health, 25, 179-181. doi:10.1016/S1054-139X(99)00023-3

[25] Langer, L.M. and Tubman, J.G. (1997) Risky sexual behavior among substance-using adolescents: Psychosocial and contextual factors. American Journal of Orthopsychiatry, 67, 315-322. doi:10.1037/h0080235 